\title{
Solutions to the Schrödinger Equation with Inversely Quadratic Yukawa Plus Inversely Quadratic Hellmann Potential Using Nikiforov-Uvarov Method
}

\author{
B. I. Ita and A. I. Ikeuba \\ Theoretical Quantum Mechanics Group, Department of Pure and Applied Chemistry, University of Calabar, \\ Calabar 00234, Nigeria \\ Correspondence should be addressed to B. I. Ita; iserom2001@yahoo.com
}

Received 20 July 2013; Revised 9 November 2013; Accepted 9 November 2013

Academic Editor: Boris A. Malomed

Copyright (C) 2013 B. I. Ita and A. I. Ikeuba. This is an open access article distributed under the Creative Commons Attribution License, which permits unrestricted use, distribution, and reproduction in any medium, provided the original work is properly cited.

\begin{abstract}
The solutions to the Schrödinger equation with inversely quadratic Yukawa and inversely quadratic Hellmann (IQYIQH) potential for any angular momentum quantum number $l$ have been presented using the Nikiforov-Uvarov method. The bound state energy eigenvalues and the corresponding unnormalized eigenfunctions are obtained in terms of the Laguerre polynomials. The NU method is related to the solutions in terms of generalized Jacobi polynomials. In the NU method, the Schrödinger equation is reduced to a generalized equation of hypergeometric type using the coordinate transformation $s=s(r)$. The equation then yields a form whose polynomial solutions are given by the well-known Rodrigues relation. With the introduction of the IQYIQH potential into the Schrödinger equation, the resultant equation is further transformed in such a way that certain polynomials with four different possible forms are obtained. Out of these forms, only one form is suitable for use in obtaining the energy eigenvalues and the corresponding eigenfunctions of the Schrödinger equation.
\end{abstract}

\section{Introduction}

The bound state solutions to the Schrödinger equation (SE) are only possible for some potentials of physical interest [15]. Quite recently, several authors have tried to solve the problem of obtaining exact or approximate solutions to the Schrödinger equation for a number of special potentials [6-10]. Some of these potentials are known to play very important roles in many fields of physics such as molecular physics, solid state, and chemical physics [8].

The purpose of the present work is to present the solution to the Schrödinger equation with the inversely quadratic Yukawa potential [11] plus inversely quadratic Hellmann potential [12] of the forms

$$
V(r)=-V_{o} \frac{e^{-2 \delta r}}{r^{2}}, \quad V(r)=-\frac{a}{r}+\frac{b}{r^{2}} e^{\delta r}, \text { respectively. }
$$

The sum of these potentials can be written as

$$
V(r)=\frac{1}{r^{2}}\left(b-V_{0}\right)+\frac{1}{r}\left(2 V_{0}-a-b \delta\right)+\left(b-2 V_{0}\right) \delta^{2},
$$

where $r$ represents the internuclear distance, $a$ and $b$ are the strengths of the Coulomb and Yukawa potentials, respectively, $\delta$ is the screening parameter, and $V_{0}$ is the dissociation energy. Equation (2) is then amenable to Nikiforov-Uvarov method. Ita [13] has solved the Schrödinger equation for the Hellman potential and obtained the energy eigenvalues and their corresponding wave functions using expansion method and Nikiforov-Uvarov method. Also, Hamzavi and Rajabi [14] have used the parametric Nikiforov-Uvarov method to obtain tensor coupling and relativistic spin and pseudospin symmetries of the Dirac equation with the Hellmann potential. Kocak et al. [15] solved the Schrödinger equation with the Hellmann potential using asymptotic iteration method and obtained energy eigenvalues and the wave functions. 
However, not much has been achieved in the area of solving the radial Schrödinger equation for any angular momentum quantum number, $l$, with IQYIQH potential using NikiforovUvarov method in the literature.

\section{Overview of the Nikiforov-Uvarov Method}

The Nikiforov-Uvarov (NU) method is based on the solutions to a generalized second-order linear differential equation with special orthogonal functions [16]. The Schrödinger equation of the type:

$$
\psi^{\prime \prime}(z)+[E-V(z)] \psi(z)=0
$$

could be solved by this method. This can be done by transforming (3) into an equation of hypergeometric type with appropriate coordinate transformation $z=z(r)$ to get

$$
\psi^{\prime \prime}(z)+\frac{\bar{\tau}(z)}{\sigma(z)} \psi^{\prime}(z)+\frac{\bar{\sigma}(z)}{\sigma^{2}(z)} \psi(z)=0 .
$$

To find the exact solution to (4), we write $\psi(z)$ as

$$
\psi(z)=\phi(z) \chi(z)
$$

Substitution of (5) into (4) yields (6) of hypergeometric type

$$
\sigma(z) \chi^{\prime \prime}(z)+\tau(z) \chi^{\prime}(z)+\lambda \chi(z)=0
$$

In (5), the wave function $\phi(z)$ is defined as the logarithmic derivative [17]

$$
\frac{\phi^{\prime}(z)}{\phi(z)}=\frac{\pi(z)}{\sigma(z)}
$$

with $\pi(z)$ being at most first-order polynomials. Also, the hypergeometric-type functions in (6) for a fixed integer $n$ are given by the Rodrigue relation as

$$
\chi_{n}(z)=\frac{B_{n}}{\rho_{n}} \frac{d^{n}}{d z^{n}}\left[\sigma^{n}(z) \rho(z)\right]
$$

where $B_{n}$ is the normalization constant and the weight function $\rho(z)$ must satisfy the condition

$$
\frac{d}{d s}\left[\sigma^{n}(z) \rho(z)\right]=\tau(z) \rho(z),
$$

with

$$
\tau(z)=\bar{\tau}(z)+2 \pi(z) .
$$

In order to accomplish the condition imposed on the weight function $\rho(z)$ it is necessary that the polynomial $\tau(z)$ be equal to zero at some points of an interval $(a, b)$ and its derivative at this interval at $\sigma(z)>0$ will be negative [17]. That is,

$$
\frac{d \tau(z)}{d z}<0
$$

The function $\pi(z)$ and the parameter $\lambda$ required for the NU method are then defined as [17]

$$
\begin{gathered}
\pi(z)=\frac{\sigma^{\prime}-\bar{\tau}}{2} \pm \sqrt{\left(\frac{\sigma^{\prime}-\bar{\tau}}{2}\right)^{2}-\bar{\sigma}+k \sigma,} \\
\lambda=k+\pi^{\prime}(z) .
\end{gathered}
$$

The $z$-values in (12) are possible to evaluate if the expression under the square root is square of polynomials. This is possible if and only if its discriminant is zero. Therefore, the new eigenvalue equation becomes [18]

$$
\lambda=\lambda_{n}=-n \frac{d \tau}{d z}-\frac{n(n-1)}{2} \frac{d^{2} \sigma}{d z^{2}}, \quad n=0,1,2, \ldots
$$

A comparison between (13) and (14) yields the energy eigenvalues.

\section{The Schrödinger Equation}

In spherical coordinate, the Schrödinger equation with the potential $V(r)$ is given as [19]

$$
\begin{aligned}
& -\frac{\hbar^{2}}{2 \mu}\left[\frac{1}{r^{2}} \frac{\partial}{\partial r}\left(r^{2} \frac{\partial}{\partial r}\right)+\frac{1}{r^{2} \sin \theta} \frac{\partial}{\partial \theta}\left(\sin \theta \frac{\partial}{\partial \theta}\right)\right. \\
& \left.\quad+\frac{1}{r^{2} \sin ^{2} \theta} \frac{\partial^{2}}{\partial \phi^{2}}\right] \psi(r, \theta, \phi)+V(r) \psi(r, \theta, \phi) \\
& =E \psi(r, \theta, \phi)
\end{aligned}
$$

Using the common ansatz for the wave function

$$
\psi(r, \theta, \phi)=\frac{R(r)}{r} Y_{l m}(\theta, \phi)
$$

in (9) we get the following set of equations:

$$
\begin{gathered}
\frac{d^{2} R_{n l}(r)}{d r^{2}}+\frac{2 \mu}{\hbar^{2}}\left[E-V(r)-\frac{\lambda \hbar^{2}}{2 \mu r^{2}}\right] R_{n l}(r)=0, \\
\frac{d^{2} \Theta_{m l}(\theta)}{d \theta^{2}}+\cot \theta \frac{d \Theta_{m l}(\theta)}{d \theta}\left(\lambda-\frac{m^{2}}{\sin ^{2} \theta}\right) \Theta_{m l}(\theta)=0 \\
\frac{d^{2} \Phi_{m}(\phi)}{d \phi^{2}}+m^{2} \Phi_{m}(\phi)=0
\end{gathered}
$$

where $\lambda=l(l+1)$ and $m^{2}$ are the separation constants. $Y_{l m}(\theta, \phi)=\Theta_{m l}(\theta) \Phi_{m}(\phi)$ is the solution to (18) and (19) and their solutions are well known as spherical harmonic functions [19].

\section{Solutions to the Radial Equation}

Equation (17) is the radial part of the Schrödinger equation which we are interested in solving. Equation (17) together 
with the potential in (2) and with the transformation $z=r^{2}$ yields the following equation:

$$
\frac{d^{2} R(z)}{d z^{2}}+\frac{1}{2 z} \frac{d R(z)}{d z}+\frac{1}{4 z^{2}}\left(-\alpha z^{2}+\beta z-\gamma\right) R(z)=0,
$$

where the radial wave function is $R(z)$ and

$$
\begin{gathered}
\alpha=-\frac{2 \mu\left(E+2 V_{o}-b\right) \delta^{2}}{\hbar^{2}}, \quad \beta=-\frac{2 \mu\left(2 V_{o} \delta-a-b \delta\right)}{\hbar^{2}}, \\
\gamma=\frac{2 \mu\left(b-V_{o}\right)}{\hbar^{2}}+l(l+1) .
\end{gathered}
$$

Equation (14) is then compared with (4) and the following expressions are obtained:

$$
\widetilde{\tau}=2, \quad \sigma(z)=z, \quad \widetilde{\sigma}=-\alpha z^{2}+\beta z-\gamma .
$$

We then obtain the function $\pi$ by substituting (22) into (12):

$$
\pi=\frac{1}{2} \pm \frac{1}{2} \sqrt{4 \alpha^{2}+(k-\beta) z+1+4 \gamma} .
$$

According to the NU method, the quadratic form under the square-root sign of (23) must be solved by setting the discriminant of this quadratic equation equal to zero; that is, $D=b^{2}-4 a c=0$. This discriminant gives a new equation which can be solved for the constant $k$ to get the two roots as

$$
k_{ \pm}=\beta \pm \sqrt{\alpha(1+4 \gamma)} .
$$

Thus, we have

$$
\begin{aligned}
& k_{-}=\beta-\sqrt{\alpha(1+4 \gamma)}, \\
& k_{+}=\beta+\sqrt{\alpha(1+4 \gamma)} .
\end{aligned}
$$

When the two values of $k$ given in (25) are substituted into (23), the four possible forms of $\pi(z)$ are obtained as

$$
\begin{aligned}
\pi(z)= & -\frac{1}{2} \\
& \pm \frac{1}{2} \begin{cases}2 \sqrt{\alpha} z+\sqrt{1+4 \gamma} & \text { for } k_{+}=\beta+\sqrt{\alpha(1+4 \gamma)} \\
2 \sqrt{\alpha} z-\sqrt{1+4 \gamma} & \text { for } k_{-}=\beta-\sqrt{\alpha(1+4 \gamma)} .\end{cases}
\end{aligned}
$$

One of the four values of the polynomial $\pi(z)$ is just proper to obtain the bound state solution since $\tau$ given in (4) must have negative derivative. Therefore, the most suitable expression of $\pi(z)$ is chosen as

$$
\pi(z)=-\frac{1}{2}-\frac{1}{2}(2 \sqrt{\alpha} z-\sqrt{1+4 \gamma})
$$

for $k_{-}=\beta-\sqrt{\alpha(1+4 \gamma)}$. We obtain $\tau(z)=1+\sqrt{1+4 \gamma}-$ $2 \sqrt{\alpha} z$ from (10) and the derivative of this expression would be negative; that is, $\tau^{\prime}(z)=-2 \sqrt{\alpha}<0$. From (19) and (20) we obtain

$$
\begin{gathered}
\lambda=\beta-\sqrt{\alpha(1+4 \gamma)}-2 \sqrt{\alpha} z, \\
\lambda_{n}=2 n \sqrt{\alpha} .
\end{gathered}
$$

When we compare these expressions, $\lambda=\lambda_{n}$, we obtain the energy of the IQYIQH potential as

$$
\begin{aligned}
E= & -\left(2 V_{o}-b\right) \delta^{2} \\
& -\frac{\mu\left(2 V_{o} \delta-a-b \delta\right)^{2} / 2 \hbar^{2}}{\left(n+(1 / 2)+\sqrt{\left(2 \mu\left(b-V_{o}\right) / \hbar^{2}\right)+(l+(1 / 2))^{2}}\right)^{2}} .
\end{aligned}
$$

Let us now calculate the radial wave function, $R(z)$. Using $\sigma$ and $\pi(7)$ and (9), the following expressions are obtained:

$$
\begin{gathered}
\phi(z)=z^{(-1+\sqrt{1+4 \gamma}) / 2} e^{-\sqrt{\alpha} z}, \\
\rho(z)=z^{\sqrt{1+4 \gamma}} e^{-2 \sqrt{\alpha} z} .
\end{gathered}
$$

Then from (8) one has

$$
\chi_{n}(z)=B_{n} z^{-\sqrt{1+4 \gamma}} e^{2 \sqrt{\alpha} z} \frac{d^{n}}{d z^{n}}\left(z^{n+\sqrt{1+4 \gamma}} e^{-2 \sqrt{\alpha} z}\right) .
$$

$B_{n}$ is a normalization constant. The wave function $R(z)$ can be obtained in terms of the generalized Laguerre polynomials as

$$
R(z)=N_{n} z^{(-1+\sqrt{1+4 \gamma}) / 2} e^{-\sqrt{\alpha} z} L_{n}^{\sqrt{1+4 \gamma}}(2 \sqrt{\alpha} z) .
$$

$N_{n}$ is the normalization constant.

\section{Discussion}

In summary, we have obtained the energy eigenvalues and the corresponding unnormalized wave function using the NU method for the Schrödinger equation with the inversely quadratic Yukawa plus inversely quadratic Hellmann potential. If we set the parameters $V_{o}=b=0$ and $a=z e^{2}$, it is easy to show that (29) reduces to the bound state energy spectrum of a particle in the Coulomb potential; that is, $E_{n p}=$ $-Z^{2} \mu e^{4} / 2 \hbar^{2} n_{p}^{2}$, where $n_{p}=n+l+1$ is the principal quantum number. Similarly, if we set $a=b=0,(29)$ results in the bound state energy spectrum of a vibrating-rotating diatomic molecule subject to the inversely quadratic Yukawa potential as follows:

$$
\begin{aligned}
E= & -\left(2 V_{o}\right) \delta^{2} \\
& -\frac{\mu\left(2 V_{o} \delta\right)^{2} / 2 \hbar^{2}}{\left(n+(1 / 2)+\sqrt{(l+(1 / 2))^{2}}-\left(2 \mu\left(V_{o}\right) / \hbar^{2}\right)\right)^{2}} .
\end{aligned}
$$

This result is similar to that obtained in [11]. Also, if we set $V_{o}=0, a \neq 0$, and $b \neq 0$, equation reduces to the bound state 
energy spectrum of a vibrating-rotating diatomic molecule subject to inversely quadratic Hellmann potential as follows:

$$
E=b \delta^{2}-\frac{\mu(a+b \delta)^{2} / 2 \hbar^{2}}{\left(n+1 / 2+\sqrt{2 \mu b / \hbar^{2}+(l+1 / 2)^{2}}\right)^{2}} .
$$

Equation (34) is also similar to the one obtained in [12]. These show the accuracy of our calculations.

\section{Conclusion}

The bound state solutions to the Schrödinger equation have been obtained for the inversely quadratic Yukawa and inversely quadratic Hellmann potential. Special cases of the potential are also considered and their energy eigenvalues are obtained.

\section{Conflict of Interests}

The authors declare that there is no conflict of interests regarding the publication of this paper.

\section{References}

[1] S. M. Ikhdair and R. Sever, "A perturbative treatment for the energy levels of neutral atoms," International Journal of Modern Physics A, vol. 21, no. 31, article 6465, 2006.

[2] A. N. Ikot and L. E. Akpabio, "Approximate solution of Schrödinger equation with Rosen-Morse potential including a centrifugal term," Applied Physics Research, vol. 2, no. 2, p. 202, 2010.

[3] B. I. Ita, "Bound state solutions of Schrödinger equation for Rydberg potential energy function," Nigerian Journal of Physics, vol. 20 , no. 2, p. 221, 2008.

[4] S. M. Ikhdair and R. Sever, "On solutions of the Schrödinger equation for some molecular potentials: wave function ansatz," Central European Journal of Physics, vol. 6, no. 3, pp. 697-703, 2008.

[5] B. I. Ita, "Any $l$-state solutions of the Schrödinger equation for a more general exponential screened coulomb potential using Maclaurin's expansion and Nikifororv-Uvarov method," International Journal of Physical Sciences, vol. 2, pp. 141-142, 2010.

[6] A. N. Ikot, "Solutions to the Klein-Gordon equation with equal scalar and vector modified Hylleraas plus exponential Rosen Morse potentials," Chinese Physics Letters, vol. 29, no. 6, Article ID 060307, 2012.

[7] A. N. Ikot, "Analytical solutions of the Schrödinger with generalized hyperbolic potential using Nikiforov-Uvarov method," The African Review of Physics, vol. 6, pp. 221-228, 2011.

[8] S. Dong and S. H. Dong, "Schrödinger equation with a coulomb field in $2+1$ dimensions," Physica Scripta, vol. 66, no. 5, aricle $342,2002$.

[9] F. Dominguez-Adame, "Bound states of the Klein-Gordon equation with vector and scalar Hulthén-type potentials," Physics Letters A, vol. 136, no. 4-5, pp. 175-177, 1989.

[10] S. H. Dong, "An algebraic approach to the harmonic oscillator plus an inverse square potential in three dimensions," The American Journal of Applied Sciences, vol. 2, no. 1, pp. 376-382, 2005.
[11] M. Hamzavi, S. M. Ikhdair, and B. I. Ita, "Approximate spin and pseudospin solutions to the Dirac equation for the inversely quadratic Yukawa potential and tensor interaction," Physica Scripta, vol. 85, no. 4, Article ID 045009, 2012.

[12] B. I. Ita, "Solutions of the Schrödinger equation with inversely quadratic Hellmann plus Mie-type potential using NikiforovUvarov method," International Journal of Recent Advances in Physics, vol. 2, no. 4, pp. 25-233, 2013.

[13] B. I. Ita, "Arbitrary angular momentum solutions of the Schrödinger equation for Hellmann potential energy function using Maclaurin's expansion and Nikiforov-Uvarov method," Ultra Science, vol. 21, pp. 573-578.

[14] M. Hamzavi and A. A. Rajabi, "Tensor coupling and relativistic spin and pseudospin symmetries with the Hellmann potential," Canadian Journal of Physics, vol. 91, no. 5, pp. 411-419, 2013.

[15] G. Kocak, O. Bayrak, and I. Boztosun, "Arbitrary l-state solution of the hellmann potential," Journal of Theoretical and Computational Chemistry, vol. 6, no. 4, article 893, 2007.

[16] A. F. Nikiforov and V. B. Uvarov, Functions of Mathematical Physics, Birkhäuser, Basel, Switzerland, 1988.

[17] C. Berkdemir, A. Berkdemir, and J. Han, "Bound state solutions of the Schrödinger equation for modified Kratzer's molecular potential," Chemical Physics Letters, vol. 417, no. 4-6, pp. 326329, 2006.

[18] L. I. Schiff, Quantum Mechanics, McGraw-Hill, New York, NY, USA, 1955.

[19] A. N. Ikot, O. A. Awoga, and B. I. Ita, "Bound state solutions of exponential-coshine screened coulomb plus morse potential," Journal of Atomic and Molecular Sciences, vol. 3, no. 4, pp. 285296, 2012. 

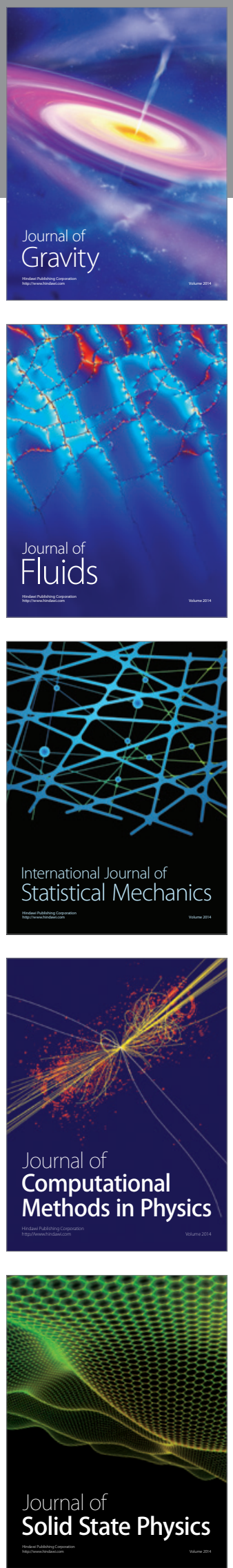

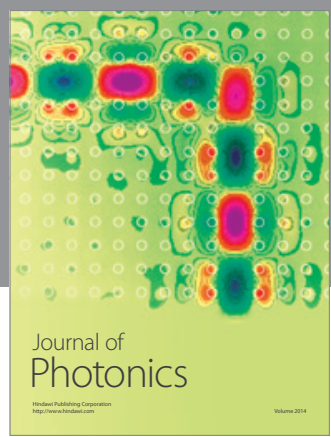

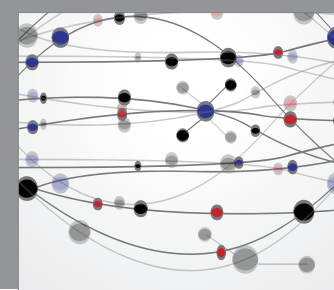

The Scientific World Journal

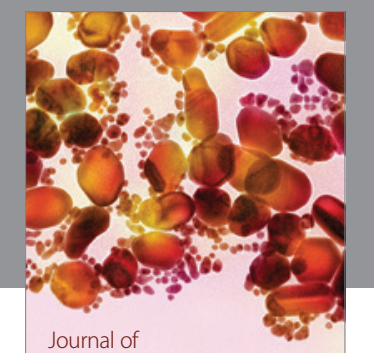

Soft Matter
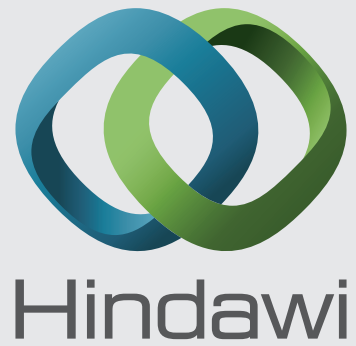

Submit your manuscripts at

http://www.hindawi.com
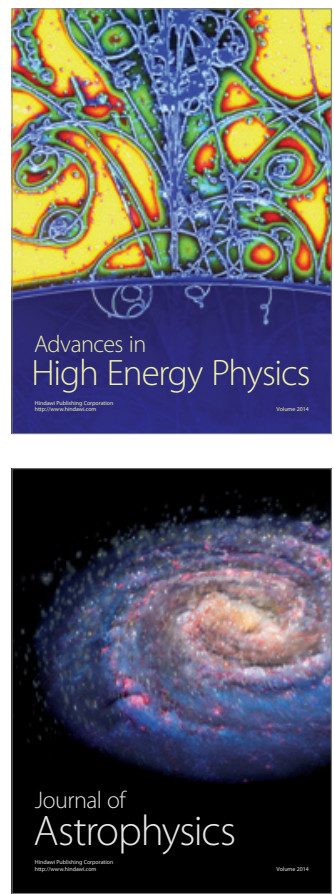
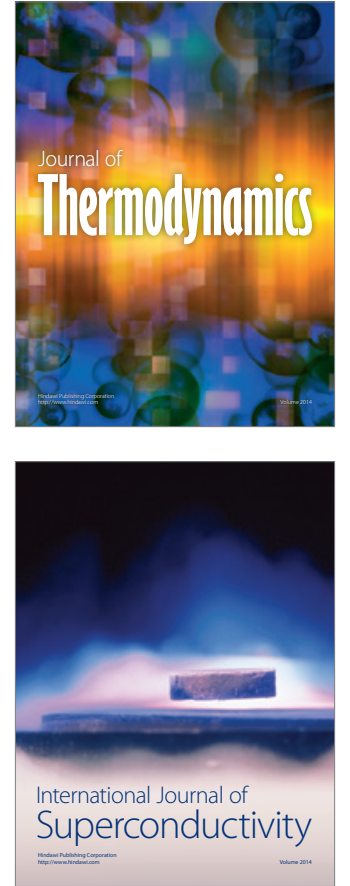
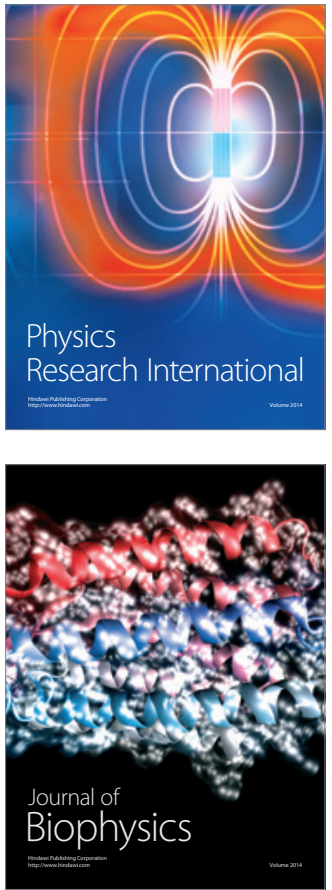
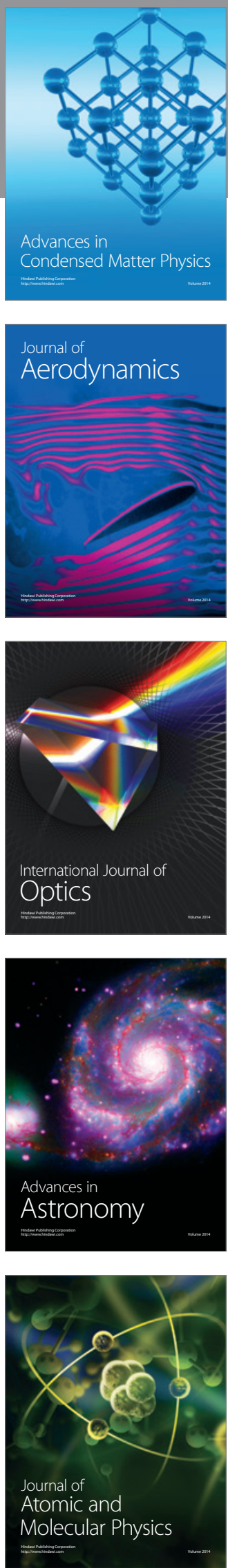